\title{
TANGENTIAL PROPERTIES OF SETS AND ARCS OF INFINITE LINEAR MEASURE
}

\section{A. S. BESICOVITCH}

The theorem on differentiability of a monotone function or, what is equivalent, on the existence of the tangent at almost all points of a rectifiable curve, is one of the most remarkable theorems of mathematics. It is remarkable because it deals with the simplest and yet fundamental notions of analysis. The theorem is deep and it had never been guessed before. It is also remarkable because it represented a fundamental tool in the rebuilding of analysis in the twentieth century. How has it happened that the theorem had not been discovered before? The problem on differentiability occupied mathematicians for something like a century before, first in attempts to prove differentiability of continuous functions and later in establishing cases of nondifferentiability. Among those who had been working we find the names of Cauchy, Riemann, Weierstrass. How is it that monotonicy had never come into consideration? But perhaps it came, and without methods of measure the problem was unsolvable? This is not true: the theory of measure is not needed for the proof. The simplest proof is not based on the theory of measure: it is proved that the set of points of a rectifiable curve at which no tangent exists can be included in a set of arcs of the curve, of arbitrarily small total length, and the set is arrived at without any use of theory of measure. The theory of measure was not needed for the proof, but the problem could not be set without ideas of measure. Riemann gave an example of a monotone function (indefinite integral) for which both the set of points of differentiability and the set of points of nondifferentiability are everywhere dense. In those days the idea that one of these sets is "almost the whole of the interval" and the other "almost empty" could not arise. As soon as Lebesgue developed the theory of measure, the theorem came as a most beautiful boon of new methods.

On the basis of $L$-measure the study of differentiability of continuous functions reached a completeness in the theorems of Denjoy, in the second decade of this century. In the same decade Carathéodory introduced methods of linear measure in planes and spaces of higher dimensions. Ideas of Carathéodory were developed by

An address delivered before the Annual meeting of the Society in Chicago on January 27, 1960 by invitation of the Committee to Select Hour Speakers for Annual and Summer Meetings; received by the editors March 26, 1960. 
Hausdorff, who considered $m$-dimensional measure in the $n$-dimensional space for any $m \leqq n$ (integral or not).

New methods of measure suggested to me a study of the geometry of sets of points which has been carried out in three papers on Fundamental Geometric Properties of Plane Sets of Points. ${ }^{1}$ The existence of a tangent at points of a rectifiable curve found its analogue in the theorem on the existence of a tangent at points of a regular set. Nonexistence was established at points of irregular sets. Just as the Lebesgue theorem was concerned with arcs of finite length, I studied sets of finite linear measure.

Among sets of infinite measure stands out the class of sets of $\sigma$-finite measure. A set $E$ of infinite measure is said to be of $\sigma$-finite measure if it can be split into an enumerable set of sets of finite measure; e.g., a straight line is of $\sigma$-finite measure.

By the nature of measure, one expects that sets of $\sigma$-finite measure have properties similar to sets of finite measure; and for the completeness of Lebesgue's theorem an answer to the question "does the theorem hold for arcs of $\sigma$-finite linear measure?" should be given.

The answer to the question is obviously in the affirmative when the arc of $\sigma$-finite linear measure can be represented as the sum of an enunerable set of arcs of finite length, but this is not a general case: an arc may be the sum of an enumerable set of sets of finite measure, which need not be arcs. There are arcs of $\sigma$-finite measure, whose every sub-arc is of infinite length.

The answer to the question in general has been given in my paper On the definition of tangent to sets of $\infty$ measure, Proc. Cambridge Philos. Soc. vol. 52 (1956). But the proof depends on all fundamental properties of linearly measurable sets, including most intrinsic properties of regular sets and irregular ones.

I shall start by recalling some definitions and some results on plane sets of finite linear measure.

Definition. Given a plane set $E$ we consider its covering by a set $U(E, \delta)$ of convex domains of diameter $d \leqq \delta$. The linear measure is defined by the equation

$$
\wedge E=\liminf _{\delta \rightarrow 0} \sum_{U(E, \delta)} d .
$$

Corollary 1. For linear sets $E, \wedge E$ coincides with the Lebesgue linear measure.

THEOREM 1. If $E$ is a plane arc, $\Lambda E$ coincides with the length of $E$, whether it is finite or not.

\footnotetext{
${ }^{1}$ Math. Ann. vol. 98 (1928); vol. 115 (1938); vol. 116 (1939).
} 
Definition. The limits of $\Lambda\{E c(M, r)\} / 2 r$, where $c(M, r)$ is the circle with center $M$ and radius $r$, as $r \rightarrow 0$, are called densities (upper, lower, the density) of $E$ at $M$ and are denoted $\bar{D}\{E, M\}, D_{*}\{\}$, $D\{\}$.

Corollary 1. If $E$ is a linear set the definition of densities coincides with the Lebesgue definition. is 1 .

COROLlary 2. If $E$ is a segment then its density at all interior points

Theorem 2. Density of $E$ at almost all points outside $E$ is 0 (that is, at all points outside $E$ with the exception of a set of linear measure 0 ).

An immediate corollary of Theorem 2 is the Lebesgue Theorem on density of linear sets.

Theorem 2 establishes invariance of densities with respect to the operations of addition and subdivision of sets:

(i) if $E=E_{1}+E_{2}$ then at almost all points $M$ of $E_{i}, i=1,2$,

$$
\bar{D}\left\{E_{i}, M\right\}=\bar{D}\{E, M\} \text {, etc. }
$$

(ii) also if $\Lambda E_{1} E_{2}>0$ then at almost all points $M$ of $E_{1} E_{2}$

$$
\bar{D}\left(E_{1}, M\right)=\bar{D}\left\{E_{2}, M\right\} \text {, etc. }
$$

(iii) given two representations of a set $E$ of $\sigma$-finite linear measure

$$
E=\sum_{i=1}^{\infty} E_{i}=\sum_{j=1}^{\infty} E_{j}^{\prime}
$$

by the sums of sets of finite measure, then at almost all points $M$ of $E$, from $M \in E_{i}, M \in E_{j}^{\prime}$ follows

$$
\bar{D}\left(E_{i}, M\right)=\bar{D}\left(E_{j}^{\prime}, M\right) \text { etc. }
$$

while for linearly measurable sets on a line the density exists and is equal to 0 or 1 at almost all points of the line. In the case of a plane set $E$ of finite linear measure we have

Theorem 3. At almost all points $M$ of $E$,

$$
\frac{1}{2} \leqq \bar{D}\{E, M\} \leqq 1 .
$$

COROLlARY. When $E$ is a rectifiable curve, then at almost all points of $E$ the density exists and is equal to 1.

Definition. A point $M$ of the set $E$ is said to be a regular point 
if the density of $E$ at $M$ exists and is equal to 1 . Any other point is an irregular one.

Definition. A set $E$ with almost all points regular is called a regular set. A set $E$ with almost all points irregular is an irregular set.

Given a set $E$ of finite linear measure, denote by $E_{1}$ the set of its regular points and by $E_{2}$ of its irregular points. Theorem 2 leads to the conclusion that $E_{1}$ is a regular set and $E_{2}$ is an irregular set, for at almost all points $M$ of $E_{1}\left(E_{2}\right)$ the densities of $E_{1}\left(E_{2}\right)$ coincide with the density of $E$. Thus

THEOREM 4. Any set of finite linear measure is the sum of a regular set and an irregular one.

The tangent to the set $E$ at a point $M$, whether belonging to the set or not, is defined as follows:

A line $l$ through $M$ is a tangent at $M$ to $E$ if (i) $\bar{D}(E, M)>0$ and (ii) for any pair $\omega$ of opposite angles with vertex $M$, that do not contain the line $l, D\{E \omega, M\}=0$.

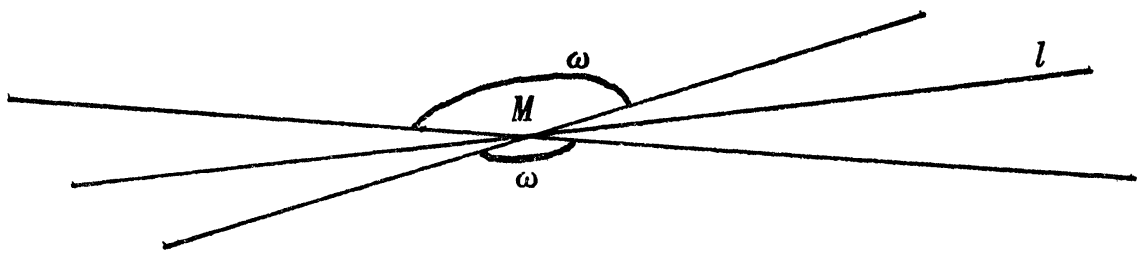

COROLlary 1. There may be at most one tangent at $M$.

Corollary 2. The definition is a generalization of the usual definition of the tangent to a rectifiable arc.

Corollary 3. Given $E=E_{1}+E_{2}$, at almost all points of $E_{1}\left(E_{2}\right)$ tangents to the sets $E$ and $E_{1}\left(E_{2}\right)$ exist simultaneously and coincide or do not exist. Thus: The general study of tangential properties of sets reduces to the study of regular sets and irregular ones.

The result is given by two theorems.

Fundamental Theorem 1. At almost all points of a regular set the tangent exists.

Fundamental Theorem 2. At almost all points of an irregular set $E$ no tangent exists and at almost all points $M$ of the set for any pair $\omega$ of opposite angles with vertex at $M$

$$
\bar{D}(E \omega, M)>0 .
$$

For the solution of our problem we need the 
Fundamental TheOREm 3. The orthogonal projection of an irregular set on almost all directions is of measure 0.

At this point it is appropriate to introduce Favard's definition of linear measure: Favard's linear measure of a set $E$ is the mean value of the linear measure of the orthogonal projection of the set on all directions. In order that this measure coincide with length when the set is a curve it is multiplied by $\pi / 2$. Then it also coincides with the Carathéodory linear measure of all regular sets. The above theorem can now be expressed in the following way:

The Favard measure of an irregular set is 0 . Then it follows from the above theorems that

At almost all points, in the Favard sense, the tangent to a set of Carathéodory finite linear measure exists.

In case of sets of infinite measure a new definition of the tangent is needed if we want the tangent to characterize some intrinsic properties of the set and not to be just a formal notion. The unsuitability of the definition we have been using up to now can be illustrated by two examples: let $C$ be the set of concentric circles of rational radii $<1$ and $L$ be the set of lines $a x+b y+c=0$ in the coordinate plane, with $a, b, c$ all rational. For each of the two sets the tangent, as defined before, does not exist, because the density of the set in any pair $\omega$ of opposite angles is $\infty$. And yet there are tangential properties in the case of each of the two sets. In fact, in the case of $C$ the tangent at $M \in C$ to the circumference containing $M$ is a strong claimant to be the tangent to $C$, and in the case of $L$ the line containing $M$ (when $M$ belongs to one line only, which is the case with all points of $L$ except an enumerable subset) is an equally strong claimant. Consider the basis of the claims. In each case the set contains a curve, or a line through $M$ and the claimant touches the curve in the case of $C$ and coincides with the line in the case of $L$. Is then the tangent at $M$ to be defined as the tangent at $M$ to a subset of finite linear measure containing $M$ ? But with this definition in the case of $C$ and $L$ every line through $M$ would be a tangent to the set at $M$. This is particularly easy to see in the case of $L$, for through every point $M$ of $L$ passes a curve totally belonging to $L$ and having an arbitrarily prescribed tangent at $M$. Such curves are polygonal lines with infinitely many sides. Yet by the nature of the sets $C$ and $L$ the above mentioned claimants remain strong ones, only a different definition of a tangent is wanted. However, the above argument shows that no useful definition of the tangent at an individual point can be given. Therefore the question is to be approached differently. Each of our sets is of $\sigma$-finite measure, that is, each one can be represented as the 
union of $\boldsymbol{\aleph}_{0}$ sets of finite measure. $C$ is defined like that and $L$ can be represented in this way by representing each line as a union of finite segments. Our claimants are tangents to the members of the union. This suggests the following general way of defining the tangents at points of a set of $\sigma$-finite measure:

Represent the set $E$ as the union of $\boldsymbol{\aleph}_{0}$ exclusive subsets of finite measure

$$
E=\sum_{i=1}^{\infty} X_{i}
$$

For every $M \in E$ we define $X_{i}$ containing $M$. If the tangent at $M$ to $X_{i}$ exists, then by definition it is the tangent to $E$ at $M$. If the tangent to $X$ at $M$ does not exist we say that the tangent to $E$ at $M$ does not exist. Thus we shall get the splitting $E=E^{\prime}+E^{\prime \prime}$ such that the tangent exists and is defined at points of $E^{\prime}$ and does not exist at points of $E^{\prime \prime}$. This method makes the existence of the tangent and its direction dependent on the way of splitting of the set $E$ into subsets of finite measure and as it can be seen from the set $L$ considered above the splitting of $E$ can be arranged so that at a fixed point the tangent has a preassigned direction. But the dependence is not so complete as it may look. In fact it is very slight. We have seen that the definition of the tangent to a set of finite measure makes the tangential properties invariant with respect to operations of addition and subdivision (just as are density values), that is, if a set $E$ of finite measure is equal to $\sum_{n=1}^{\infty} E_{n}$ than at almost all points $M$ of $E$ the tangential properties of $E$ coincide with those of $E_{n}$ containing $M$. Let now $E$ be a set of $\sigma$-finite measure and $\sum_{n=1}^{\infty} X_{n}, \sum_{m=1}^{\infty} Y_{m}$ be two different representations of $E$ as sums of sets of finite measure. Writing $X_{n}$ $=\sum_{m=1}^{\infty} X_{n} Y_{m}$, we see that at almost all points $M$ of $X_{n}$ the tangential properties of $X_{n}$ coincide with those of $X_{n} Y_{m}$ containing $M$. This being true for any $n$ we conclude that at almost all points of $E$ the tangential properties defined by the subdivision $E=\sum X_{n}$ coincide with the tangential properties defined by the subdivision $E=\sum_{n, m} X_{n} Y_{m}$. Similarly the tangential properties defined by the subdivision $\sum Y_{m}$ coincide with those defined by the subdivision $\sum_{n, m} X_{n} Y_{m}$ and thus

At almost all points of $E$ the tangential properties defined by any pair of subdivisions coincide.

Given a set $E$ of $\sigma$-finite measure and a subdivision $E=\sum_{1}^{\infty} X_{n}$ into subsets of finite measure we write $X_{n}=X_{n}^{\prime}+X_{n}^{\prime \prime}$, where the $X_{n}^{\prime}$ is the regular component of $X_{n}$ and $X_{n}^{\prime \prime}$ the irregular one. The set $\sum_{n=1}^{\infty} X_{n}^{\prime}$ is by definition a regular component of $E$, and the set 
$\sum X_{n}^{\prime \prime}$ an irregular one. The set of tangents to $X_{n}^{\prime}$ for all $n$ is called a tangent set of $E$. The tangent sets of $E$, corresponding to two different subdivisions, may differ only at a set of measure 0 .

Given an arbitrary subset $X$ of finite measure, at almost all points of $X$ at which tangents to $X$ exist they coincide with those of the tangent set. Thus any tangent set gives a complete description of tangential properties of the set, and if it remains indetermined at a set of points of measure 0 , it is due not to failure of the method, but to the nature of the problem.

We are now in a position to answer the original question on tangential properties of an arc of infinite length and of $\sigma$-finite measure. Let $E$ be such an arc and $E^{\prime}$ and $E^{\prime \prime}$ its regular and irregular components respectively. It follows from the Fundamental Theorem 3 that the orthogonal projection of $E^{\prime \prime}$ on almost all direction is of measure 0 . A polygonal line $A_{0} A_{1} \cdots A_{n}$ can be inscribed into $E$, so that its sides be as small as we please, and their directions be such that the orthogonal projection of $E^{\prime \prime}$ on each of them be of measure zero. The arc $E$ is divided into sub arcs $-A_{i} A_{i+1}, i=0,1, \cdots, n-1$. The orthogonal projection of the arc $\smile A_{i} A_{i+1}$ on the chord $A_{i} A_{i+1}$ contains the whole of the chord. As the projection of $E^{\prime \prime} \smile A_{i} A_{i+1}$ on $A_{i} A_{i+1}$ has measure 0 the projection of $E^{\prime} \smile A_{i} A_{i+1}$ is of measure $\geqq \operatorname{chord} A_{i} A_{i+1}$. Hence

$$
\wedge E^{\prime} \smile A_{i} A_{i+1} \geqq \operatorname{chord} A_{i} A_{i+1}
$$

and

$$
\bigwedge E^{\prime} \geqq A_{0} A_{1} \cdots A_{n},
$$

that is,

$$
\Lambda E^{\prime}=\infty .
$$

Thus the set of points at which the tangent to $E$ exists is of infinite measure and the set of points at which it does not exist is projected into a set of measure zero on almost all directions, that is, is of Favard measure 0: The Lebesgue Theorem: At almost all points of an arc of finite length the tangent exists holds for arcs of $\sigma$-finite measure with the difference that "almost all points" is meant in the sense of Favard measure.

Cambridge University, England and University of Pennsyluania 